\title{
Serviço de atendimento psicológico especializado aos imigrantes e refugiados: interface entre o social, a saúde e a clínica
}

\author{
Specialized psychological aid service for immigrants \\ and refugees as a model for social, health \\ and clinical integration
}

\author{
Lucienne MARTINS BORGES ${ }^{1}$ \\ Jean-Bernard POCREAU
}

\begin{abstract}
Resumo
Com as novas reflexões sobre o tema da saúde e a evolução do espaço ocupado pela psicologia nas instituições públicas, viu-se necessário repensar a psicologia, seu método, sua teoria e sua prática, para melhor responder às novas exigências sociais. 0 objetivo principal deste trabalho foi apresentar o Serviço de Atendimento Psicológico Especializado aos Imigrantes e Refugiados, cujas atividades acontecem no Departamento de Psicologia da Universidade Laval, Québec, Canadá. Esse serviço surgiu de uma preocupação dos dirigentes governamentais de órgãos da saúde e dos serviços sociais, dos profissionais que atuavam nas instituições públicas e de especialistas em imigração, no que se referia à acessibilidade e à adequação dos serviços da saúde para a população de imigrantes refugiados. As atividades do Serviço de Atendimento Psicológico Especializado aos Imigrantes e Refugiados encontram-se em seu décimo ano.
\end{abstract}

Unitermos: Psicologia da saúde. Psicologia intercultural. Refugiados.

\begin{abstract}
Recent reflections on public health and the changing role of psychology within public institutions have led to a perceived need to re-think psychology with regard to its methods, theories, and practice, so as to better adapt psychology to newly emerging social needs. The objective of this study is to present the Service d'Aide Psychologique Spécialisée aux Immigrants et Réfugiés, active for ten years now at l'École de Psychologie, Université Laval, Canada. This service was created in response to preoccupations expressed by directors of public health and social service institutions, by professionals working in public institutions and by immigration specialists, all of whom questioned the accessibility and adequacy of services offered to refugee immigrants. The service is supported by grants from the Ministère de la Santé du Québec and by the United Nations.
\end{abstract}

Uniterms: Health psychology. Cross-cultural psychology. Refugees.

Desde as novas reflexões, nos anos 1960 e em várias regiões do mundo, acerca dos temas ligados à saúde, as políticas públicas evoluíram em direção à perspectiva de enfatizar uma maior valorização da

$\boldsymbol{\nabla \nabla \nabla \nabla}$

1 Universidade Federal de Santa Catarina, Centro de Filosofia e Ciências Humanas, Departamento de Psicologia. Campus Universitário. Trindade, 88040-970, Florianópolis, SC, Brasil. Correspondência para/Correspondence to: L. MARTINS BORGES. E-mail: <lucienne@cfh.ufsc.br>.

2 Université Laval, Faculté des Sciences Sociales, École de Psychologie. Québec, Canada. 
qualidade de vida das pessoas (Brasil, 2002), superando as orientações centradas unicamente no controle da doença. Ao descentralizar do campo das ciências médicas a atenção à saúde, a psicologia desenvolveu-se como uma disciplina que passou a contribuir de forma eficaz no tratamento das doenças e na promoção da saúde. Ao abrir espaço para a prática psicológica nas instituições públicas, novas preocupações surgem. Como indicado por Dimenstein (1998), como atingir resultados satisfatórios nas intervenções psicológicas realizadas em contextos e com populações que diferem daquelas já conhecidas pela psicologia? Como transpor o modelo tradicional de intervenção e a universalidade das teorias ensinadas, pensadas principalmente para a prática privada e a saúde mental, a realidades sociais complexas e às novas demandas sociais? Foi precisamente no intuito de tentar contornar tais dificuldades, e com o objetivo de oferecer um atendimento especializado e satisfatório (acessibilidade e resultados), que se deu início à elaboração do Serviço de Atendimento Psicológico Especializado aos Imigrantes e Refugiados Service d'Aide Psychologique Spécialisée aux Immigrants et Réfugiés (SAPSIR).

O SAPSIR constitui uma clínica de atendimento psicológico cujas atividades são realizadas na Universidade Laval, na cidade de Québec, Canadá. É uma clínica interdisciplinar, especializada no acolhimento de imigrantes refugiados, que foram submetidos aos mais variados tipos de violência (guerras, genocídios, torturas etc.), criada em 2000, por iniciativa de vários setores: educação (Departamento de Psicologia da Universidade Laval), saúde (Agência da Saúde e dos Serviços Sociais da Capital Nacional) e social (Organizações Não-Governamentais - ONG e associações).

Nesse sentido, pode-se considerar que esse serviço - cuja estrutura será mais relatada posteriormente com mais detalhe - se inscreve na interface de várias disciplinas, de várias instâncias governamentais (educação, saúde e serviços sociais) e de várias áreas da psicologia (clínica, social, saúde e comunitária). O objetivo principal do presente trabalho é expor o percurso desse serviço de atendimento psicológico, o SAPSIR, inscrevendo-o no âmbito de duas disciplinas centrais da 578 Psicologia: a Psicologia da Saúde e a Psicologia Clínica.

\section{A saúde e a doença}

A psicologia da saúde, através do espaço que essa disciplina ocupa no campo da psicologia e em relação a outras disciplinas, representa uma nova orientação que visa integrar as dimensões psicológicas e sociais na compreensão da saúde e da doença. De acordo com Fischer e Tarquínio (2002, p.1), “a psicologia da saúde constitui uma nova forma de abordar e de compreender os problemas da saúde e da doença". O progresso dessa disciplina dá-se paralelamente a uma tentativa atual de apreensão do comportamento individual e coletivo através da atenção direcionada aos comportamentos da saúde (Fischer, 2002; Dimenstein, 1998). Tal esforço de compreensão é realizado pelas mais diversas disciplinas eáreas: Educação, Psicologia, Ciências Sociais, Medicina etc. (Fischer \& Tarquinio, 2002).

Na sociedade ocidental, a saúde não é mais vista como um objeto médico, mas sim como um fenômeno social, que se traduz por um conjunto de atitudes que visam à promoção do bem-estar físico, mental e social. As preocupações relativas à saúde evoluíram de acordo com as doenças que caracterizam cada época, com a evolução dos quadros patológicos (das doenças infecciosas no início do século XX à predominância, no século XXI, de doenças como o câncer), mas igualmente - e sobretudo - de acordo com as políticas públicas (Fischer \& Tarquinio, 2002). As políticas públicas são o resultado de movimentos e reivindicações sociais que também evoluíram e constituem o reflexo dos momentos sociais e políticos (Dimenstein, 1998).

Apesar da evolução da compreensão da saúde, da evolução tecnológica no tratamento das doenças (que permitiu a maior longevidade das pessoas e a transformação das doenças epidêmicas em crônicas), até os anos de 1980, os procedimentos de prevenção, avaliação e ação organizavam-se em torno dos hospitais. Por conseguinte, o olhar sobre a saúde (para não dizer sobre as doenças) era predominantemente biológico, pouco atento aos fatores psicológicos e psicossociais (Spink, 2007). O confinamento dos tratamentos de saúde em instituições hospitalares fez com que a concepção dominante de saúde se mantivesse centrada sobre o bem-estar físico, em detrimento do bem-estar psicológico e global da pessoa. 
Atualmente, com o avanço das pesquisas médicas, novos conhecimentos foram adquiridos no que se refere ao desenvolvimento das doenças, aumentando a expectativa de vida. Paralelamente ao progresso científico, a introdução de novas dimensões para a compreensão das relações entre o psíquico e o corpo acarretaram grandes mudanças nas ciências humanas e sociais. Esse movimento se deu como tentativa de adaptação de um modelo universal de intervenção - como o modelo ensinado e aplicado pela psicologia -, a fim de adquirir novos métodos de intervenção que pudessem proporcionar resultados mais satisfatórios (Dimenstein, 1998).

É certo que essa relação já se encontrava presente nas teorias psicológicas, principalmente psicossomáticas, desde as primeiras preocupações de Freud (1893-1895/1988) no que se refere ao que ele denominava de conversões histéricas. Mas o termo saúde, na psicologia, permaneceu durante muito tempo relacionado apenas à saúde mental, com uma concepção da saúde psíquica que se apoiava em uma definição do funcionamento psíquico em termos de equilíbrio ou de psicopatologia (Fischer \& Tarquínio, 2002). Nas três últimas décadas, viu-se uma expansão na tentativa de compreensão da saúde e da doença - por parte das disciplinas concernidas - como sendo o resultado de vários fatores interdependentes, biológicos, psicológicos e sociais, que podem manter o indivíduo em estado de saúde ou podem constituir os desencadeadores de uma doença. Foi nesse contexto científico que se deu o nascimento e o desenvolvimento da psicologia da saúde.

\section{Psicologia da saúde: considerações atuais}

A psicologia da saúde propõe uma nova leitura da saúde, não mais exclusivamente da saúde mental, mas no sentido de que os fatores psicológicos são apreendidos como elementos inerentes ao bem-estar físico propriamente dito (Ronzani \& Rodrigues, 2006; Fischer, 2002). A disciplina tem como objetivo "compreender como os fatores biológicos, comportamentais e sociais influenciam na saúde e na doença" (Castro, 2004, p.49). Os fatores psicológicos determinam, assim, a saúde, compreendida como um estado global de bem-estar físico e mental. Saúde física e saúde mental não constituem mais duas entidades separadas, mas processos dinâmicos nos quais o biológico e psicológico interagem ininterruptamente.

A psicologia da saúde é uma disciplina relativamente recente. Criada em 1979 pela Associação Americana de Psicologia, suas principais orientações foram definidas em 1985 (Fischer \& Tarquínio, 2002; Castro, 2004). Ela se constituiu como uma área específica da psicologia e se desenvolveu, inicialmente, no contexto norte-americano, como disciplina científica mais voltada para a pesquisa. Porém, mesmo tendo sido pensada a partir da pesquisa, a psicologia da saúde constitui uma área de intervenção e de prática para a psicologia. Ela estendeu a prática clínica da psicologia para as instituições de saúde, como os hospitais, os centros de saúde, os ambulatórios etc., e integrou-a nas práticas das equipes. Em 1986, com a criação da European Health Psychology (Castro, 2004), iniciou-se a consolidação da disciplina na Europa. Por sua vez, a América Latina dotou-se de sua primeira associação, a Associação Latino-Americana de Psicologia da Saúde (ALAPSA), em 1993.

Enquanto disciplina, a psicologia da saúde estuda os diferentes fatores (psicológicos, sociais e biológicos) que intervêm na manutenção da saúde ou no desencadeamento e evolução das doenças. A saúde, nesse contexto, é apreendida globalmente e considerada a partir das diferentes situações de vida (desenvolvimento afetivo, familiar, no grupo social etc.) que afetam a integridade tanto física quanto psicológica da pessoa. Ela enfatiza, igualmente, os recursos psíquicos e a responsabilidade de cada indivíduo em assegurar seu próprio bem-estar e considera os impactos dos fatores (predicadores e moderadores) psicossociais na saúde (Fischer, 2002; Fischer \& Tarquínio, 2002). Segundo os autores, quanto aos predicadores, eles podem assumir o papel de antecedentes ou desencadeadores (como os fatores situacionais ou características da personalidade); quanto aos fatores moderadores, eles se referem ao conjunto dos processos cognitivos e emocionais que podem tornar mais grave ou diminuir o impacto de situação estressante. Assim, a psicologia da saúde organiza-se em torno da prevenção e da promoção da saúde, dos estilos de vida sadios, das situações - individuais e coletivas - envolvendo a doença, dos comportamentos adotados pelos indivíduos doentes e das consequências da doença sobre o estado geral de saúde do indivíduo. 
No que se refere à prevenção e promoção de comportamentos e estilos de vida, a psicologia da saúde interessa-se pelos comportamentos de risco que podem constituir a causa direta de certas doenças (cânceres, diabetes, doenças cardiovasculares, Human Immunodeficiency Vírus - HIV etc.) e pelas ações preventivas dessas doenças (pela promoção da saúde e de estilos de vida mais sadios). No que se refere às situações ligadas diretamente à doença, resumidamente, a psicologia da saúde interessa-se pelas condições de tratamento terapêutico realizado pelos profissionais das equipes multidisciplinares, pela relação estabelecida entre eles e o paciente, pelos modos de comunicação e transmissão das informações, pelas tomadas de decisão por parte dos profissionais e pelas atitudes do paciente face às recomendações recebidas. Por fim, no que se refere aos comportamentos adotados pelos pacientes, a psicologia da saúde preocupa-se com os aspectos psicológicos da experiência da pessoa doente, isto é, as reações à dor, as estratégias de adaptação, a qualidade de vida (grupo social, autonomia etc.) (Fischer, 2002; Castro, 2004; Fischer \& Tarquínio, 2002). Para isso, a psicologia da saúde apoia-se em uma perspectiva multifatorial, em uma dimensão biopsicossocial na qual as variáveis psíquicas, sociais e biológicas compõem um sistema de interação que assume papel determinante na saúde e na doença (Fischer, 2002; Castro, 2004).

\section{Dimensão biopsicossocial}

A psicologia da saúde desenvolveu-se através de uma abordagem influenciada pela perspectiva de integração e articulação entre várias disciplinas (Psicologia Social, Psicologia Clínica, Biologia, Neurociências etc.), a fim de compreender e explicar os diferentes fatores - biológicos, psicológicos e sociais - que interagem nos comportamentos ligados à saúde.

O método biopsicossocial é o modelo teórico de referência que corresponde de forma mais adequada à noção de saúde adotada pela psicologia da saúde, diferentemente do modelo médico, que apreende a doença a partir de desequilíbrios orgânicos. Ela considera que os fatores psicológicos, biológicos e sociais têm - individualmente e de forma interdependente - uma ação

580 determinante sobre a saúde e a doença, em que as dimensões biológica e psíquica encontram-se diretamente relacionadas uma com a outra (Fischer \& Tarquínio, 2002). Para a psicologia da saúde, o método biopsicossocial, além de ser um modelo teórico, constitui um tipo de prática no contexto clínico. O modelo apoia-se em uma prática interdisciplinar, que avalia os diferentes fatores que impactam o estado de saúde do indivíduo, considerando-o em sua globalidade (individual e social) e preocupando-se em tornar o tratamento coerente com suas particularidades e necessidades (Costa, 2007). Todas essas dimensões são consideradas de forma concomitante com a atenção prestada à relação profissional-paciente, elemento essencial da eficácia terapêutica. Assim, Castro (2004, p.50) sublinha que a psicologia da saúde "fundamenta seu trabalho principalmente na promoção e na educação para a saúde... . O trabalho é multiplicador, uma vez que capacita a própria comunidade para ser o agente da transformação".

Considerando o método, a abordagem, os objetivos e a compreensão propostos pela psicologia da saúde, pode-se afirmar que essa disciplina muito contribuiu para a participação da psicologia nas problemáticas sociais e suas novas demandas. A inserção da psicologia da saúde no campo da saúde, sua proposta de compreensão global do indivíduo, faz com que a psicologia possa agir tanto no tratamento e na prevenção, quanto na doença e na saúde.

\section{OServiço de Atendimento Psicológico Especializado aos Imigrantes e Refugiados}

O Serviço de Atendimento Psicológico Especializado aos Imigrantes e Refugiados, cujas atividades são realizadas desde 2000 na Clínica de Psicologia do Departamento de Psicologia da Universidade Laval, em Québec, Canadá, é o fruto de reflexões acerca da importância da codificação cultural na expressão e estruturação do sofrimento psicológico de um grande número de imigrantes e refugiados. O projeto de criação do SAPSIR - apesar de já fazer parte dos interesses profissionais de seus fundadores - foi lançado pela Agência da Saúde e dos Serviços Sociais da Capital Nacional (ao longo do texto será denominado apenas Agência), na cidade de Québec. A Agência, representante adminis- 
trativo regional do Ministério da Saúde e dos Serviços Sociais (Ministère de la Santé et des Services Sociaux du Québec - MSSS, n.d.), tem por missão geral criar, em seu território, uma organização de serviços integrados a fim de torná-los mais próximos da população e facilitar assim o encaminhamento de todas as pessoas dentro da rede de serviços. Em decorrência dessa política de oferta de serviços à população, a Agência estava cada vez mais preocupada com as dificuldades encontradas nos serviços oferecidos aos imigrantes e mais particularmente aos imigrantes/refugiados, isto é, aqueles que por motivos humanitários encontram-se em território canadense. Assim, foi essencialmente a partir dessa preocupação - da acessibilidade e da qualidade dos serviços de saúde oferecidos à população de refugiados da cidade de Québec - que se elaborou oficialmente o pedido de criação do SAPSIR.

Entre os elementos preponderantes na criação dessa clínica especializada está o aumento do número de imigrantes permanentes no Estado do Québec, território de abrangência do SAPSIR. Com o aumento do efetivo de imigrantes, os dados gerais que caracterizam a imigração, como o multiculturalismo, as contribuições à sociedade de acolhimento etc., cresceram e diversificaram-se. Porém, não são apenas os efeitos positivos desse aumento que se tornaram perceptíveis, mas também tudo o que, diretamente ou não, tem um impacto sobre o bem-estar psicológico dos imigrantes. $\bigcirc$ Estado do Québec tem uma população de aproximadamente 7500000 habitantes, sendo que 12,2\% são representados pela população imigrante. Entre 2001 e 2006, o crescimento da população imigrante foi de 20,5\%, ao passo que o crescimento da população quebequense como um todo foi de 4,4\% (Turcotte, 2009), o que constitui um dado significativo da evolução do movimento migratório e de suas implicações no território quebequense. Considerando apenas a cidade de Québec - onde são realizadas as atividades do SAPSIR -, em média 2 mil imigrantes aí se instalam anualmente (Ville de Québec, 2009). Quase 50,0\% são refugiados vindos da Colômbia, Congo, Ruanda, Burundi, ex-lugoslávia e países vizinhos desses citados.

Em razão do aumento do contingente de refugiados, e da vulnerabilidade desse grupo de imigrantes que chegavam diretamente de zonas de conflito ou de campos de refugiados, cresceu significativamente a demanda de cuidados formulada diretamente por eles e pelas ONG que os acolhem. Em consequência, os profissionais da rede de saúde e dos serviços sociais encontravam-se cada vez mais confrontados com casos cuja sintomatologia estava estreitamente ligada e expressa através de uma forte codificação cultural. À medida que o paciente expressava seu sofrimento por meio de representações e significações pertencentes à cultura que o constituiu, uma distância instalava-se entre ele e o profissional - igualmente codificado culturalmente, mas não pelo mesmo quadro cultural. Os comitês institucionais registravam, assim, um aumento da evasão dos pacientes e uma baixa eficácia das intervenções. Os pacientes queixavam-se de que não conseguiam fazer-se entender. Foi precisamente a partir dessa preocupação, em torno do mal-entendido entre dois referentes culturais, que se concretizou a demanda de criação do SAPSIR.

As atividades do SAPSIR são quase todas realizadas com pessoas vítimas de guerra e de tortura, sendo esses seus critérios de referência. Os pacientes são encaminhados por profissionais da rede de saúde e serviços sociais, devido à forte codificação cultural presente em sua expressão de sofrimento psicológico. É nessas pessoas que se encontram perceptíveis os efeitos mais nefastos, no plano psicológico, do processo migratório; efeitos quase sempre relacionados com as perdas (do país, das pessoas, do referencial cultural, da identidade etc.) e, por conseguinte, com o luto.

\section{Acessibilidade}

A Lei Sobre os Serviços de Saúde e os Serviços Sociais (LSSSS) do Estado do Québec estabelece os grandes princípios legais atinentes aos serviços oferecidos à população: as linhas diretrizes, o direito aos serviços adequados e o direito de escolher o profissional. Tal Lei constitui elemento essencial para o reconhecimento da acessibilidade dos imigrantes aos serviços de saúde e aos serviços sociais (Tremblay, 2006).

Em decorrência das políticas de imigração dos anos 90, que visavam ao aumento do contingente de imigrantes e à melhoria de sua qualidade de vida, o MSSS do Québec adotou, em 1989 (plano revisto em 1994), novas orientações e um plano de ação no que concerne à acessibilidade da comunidade etnocultural 
aos serviços. Em 1992, foi criado o Comitê consultativo sobre a acessibilidade e a adaptação dos serviços de saúde e dos serviços sociais e psicossociais oferecidos às pessoas oriundas de comunidades etnoculturais para a região da cidade de Québec, a fim de observar a evolução dos serviços oferecidos a essa população. Foi nesse contexto que se propôs o SAPSIR como um serviço alternativo.

Em 2003, com a adoção da Lei sobre as agências de desenvolvimento de redes locais de serviços de saúde e de serviços sociais, ocorreram mudanças significativas na organização das redes de serviços que visavam diminuir a distância entre eles e a população, assim como facilitar a mobilidade dentro da rede. Em 2005, a LSSSS foi modificada, atribuindo maior autonomia aos Centros de Saúde e de Serviços Sociais (CSSS), representantes locais da Agência e porta de acesso ao sistema de saúde como um todo, reafirmando sua responsabilidade em relação aos projetos clínicos voltados à população. A oferta dos serviços terá, então, que levar em consideração as características culturais e linguísticas das comunidades etnoculturais (Tremblay, 2006).

Foi nesse contexto que o SAPSIR se inscreveu no plano trienal da Agência, como uma entidade relacionada à categoria "Outros colaboradores: serviços oferecidos pelas organizações comunitárias dedicadas às comunidades etnoculturais em saúde e serviços sociais" (Tremblay, 2006, p.14). Apesar de constituir uma entidade distinta dos organismos comunitários, o SAPSIR foi considerado um serviço de mediação entre a população e os demais serviços públicos e comunitários. O SAPSIR acompanhou, durante esses últimos 10 anos, as mudanças ocorridas no sistema de saúde e de serviços sociais e soube adaptar-se a elas. Além disso, por meio da participação de seus membros nos mais diversos comitês diretamente ligados às questões de saúde, o SAPSIR pôde contribuir para as modificações realizadas pelo setor da saúde pública no que se refere à acessibilidade e adequação de novos serviços à população. Em 2005, o SAPSIR foi reconhecido e subsidiado pela ONU como um dos sete serviços canadenses que oferecem atendimento psicológico às vítimas diretas e indiretas de tortura.

As pessoas que necessitam de atendimento são encaminhadas ao SAPSIR pelos profissionais da saúde e dos serviços sociais (hospitais, centros de saúde, 582 clínicas externas, ONG etc.). Quando o profissional que encaminha o imigrante ao SAPSIR está disponível, solicita-se sua presença no primeiro encontro, a fim de tranquilizar o paciente e validar sua confiança em uma situação na qual, uma vez mais, ele deverá expor sua história. Esse acompanhamento tem por objetivo transferir a confiança já adquirida pelo profissional e, por meio dele, estabelecer uma mediação entre o paciente e a equipe do SAPSIR. Essa mediação - elemento essencial do trabalho - é a primeira estabelecida no contexto da clínica intercultural que constitui a prática do SAPSIR.

\section{Mediação}

Pode-se dizer que o SAPSIR é uma clínica do exílio e da migração, uma clínica do vínculo e da mediação, de um espaço transicional, no qual é exercida uma atividade terapêutica, dispensada por uma equipe de profissionais (psicólogos, assistentes sociais, médicos, antropólogos) e doutorandos em psicologia, geralmente de origem cultural diferente e que falam, no mínimo, sua língua materna e o francês (língua oficial do Québec). Esse espaço transicional, também considerado um espaço de mediação e de moderação, estabelece o contato entre várias culturas diferentes, principalmente no que se diz respeito às mais diversas concepções da saúde e da doença, e de sua etiologia e tratamento (Martins Borges \& Pocreau, 2009).

No que se refere à mediação, o trabalho do SAPSIR dá-se em três níveis, propiciando a mediação entre as diversas áreas e disciplinas implicadas em seu dispositivo clínico, entre o paciente e as instituições da saúde e serviços sociais, entre o País natal e o País de acolhimento. A mediação entre as disciplinas faz-se através do trabalho interdisciplinar, com o objetivo comum de compreensão e mudança da situação da pessoa em prol de seu bem-estar. A mediação entre os profissionais do SAPSIR e o meio externo (representado pelas diversas instituições de saúde: hospitais, centros de saúde, clínicas externas) e de serviços sociais (ONG, associações, serviços de proteção da juventude etc.) dá-se com os contatos permanentes com os profissionais dessas instituições (encaminhamentos feitos ao SAPSIR e pelo SAPSIR) e das participações em comitês institucionais. A mediação entre os dois mundos - o País natal e o País de acolhimento - dá-se pela importância e pelo lugar privilegiado atribuídos aos conteúdos culturais na expressão e na compreensão das manifestações da saúde e do sofrimento. 


\section{O dispositivo clínico: interdisciplinaridade}

O modelo teórico de intervenção do SAPSIR apoia-se na teoria de Georges Devereux (Devereux, 1967; 1972), desenvolvida em torno do postulado da universalidade do funcionamento psíquico. O autor apresenta uma forma original e determinada de considerar o material cultural como um poderoso apoio terapêutico, suscetível de desencadear associações de ideias e de ativar processos mentais bloqueados pela perda do quadro cultural e pelos traumatismos pré e pós-migratórios.

O dispositivo clínico do Sapsir foi inspirado no modelo da etnopsiquiatria, tal qual praticada na Europa e na América do Norte (Martins Borges \& Pocreau, 2009), proposto por Tobie Nathan (Nathan, 1986; 1994) e Moro (1998). Moro (1998, p.38), foi retida a constatação de que "se todo homem tende ao universal, ele tende ao universal através do que é específico à sua cultura de afiliação". de Nathan, o dispositivo de grupo pela sua função de "envelope" e de multiplicador da alteridade.

As intervenções são realizadas por um grupo de profissionais (psicólogos, assistentes sociais, médicos etc.) que são, em sua maioria, igualmente imigrantes. A língua materna é valorizada, pois ela é considerada um modo importante da expressão da afetividade (Mesmin \& Bâ, 1995). Quando se mostra necessário, solicita-se a colaboração de um mediador cultural (chamado "intérprete"), que garante não apenas a tradução da língua, mas também a da cultura (Martins Borges \& Pocreau, 2009). É importante favorecer a expressão da experiência vivida e dos afetos na língua materna - língua de formação simbólica e dos primeiros vínculos afetivos - e consagrar um grande espaço aos dados culturais e às representações sociais.

Os profissionais do SAPSIR trabalham em grupo de terapeutas (coterapias, com grupos de dois ou mais terapeutas), dentro de um dispositivo específico, no qual uma atenção particular é dada à dimensão cultural do transtorno e de sua expressão sintomática, sem negligenciar, no entanto, a análise dos funcionamentos psíquicos. Para que o atendimento desses pacientes seja possível, o trabalho requer, da parte do profissional, uma adaptação de seu modo de pensar a saúde e a doença (Sturm, 2004). É precisamente para que se torne possível um encontro entre universos de pensamentos e de lógicas culturais diferentes, para que seja viável o estabelecimento de uma relação operante, que a etnopsiquiatria se apoia em tal dispositivo, que proporciona o estabelecimento de vínculos múltiplos entre os profissionais, entre o paciente e cada profissional, e entre o paciente e o mediador cultural (intérprete) que o acompanha (Martins Borges \& Pocreau, 2009).

As intervenções clínicas do SAPSIR visam aos seguintes objetivos gerais: 1) reduzir os sintomas clássicos do sofrimento psicológico (ansiedade, afetos depressivos, sentimentos de perda e luto, transtornos somáticos diversos etc.); 2) reduzir as manifestações sintomáticas do Transtorno de Estresse Pós-Traumático (TEPT) (pesadelos, sobressaltos, angústia, pavor, imagens do evento traumatizante etc.); 3) identificar e prevenir o agravamento das situações problemáticas de risco; 4) restaurar as forças e os mecanismos de adaptação da pessoa e dos membros de sua família (reorganização da dinâmica intrafamiliar) e 5) estimular os vínculos da pessoa com seu meio psicossocial e apoiá-la em suas dificuldades de adaptação. O SAPSIR age igualmente como consultor para profissionais das próprias instituições de encaminhamento, a fim de contribuir no atendimento feito por eles próprios.

\section{Perfil dos pacientes}

De 2000 a 2008, pessoas de mais de 30 países diferentes foram atendidas (atendimento psicológico/ psicoterapia) no SAPSIR, com uma predominância de pessoas vindas da ex-lugoslávia, dos países africanos dos Grandes Lagos (Burundi, República Democrática do Congo, Ruanda, passando pelos campos de refugiados no Quênia e Tanzânia), da Colômbia e do Afeganistão. Foram realizados atendimentos individuais (criança, adolescente, adulto), bem como de casais e famílias. A taxa de abandono e de ausência é significativamente menor do que aquelas apresentadas pelos outros serviços de atendimento psicológico. No SAPSIR, 78\% dos atendimentos realizados chegaram ao fim, contrastando com os dados da rede pública, em que, em média, 50\% dos pacientes imigrantes abandonam o tratamento depois do segundo encontro (Streit, 1996). Os sintomas depressivos e do TEPT foram os mais frequentemente observados, acompanhados de angústia, 
ansiedade, irritabilidade, conflitos familiares e dificuldades de adaptação. As pessoas que consultam o SAPSIR são refugiados, vítimas de guerras e de perturbações violentas que marcam sua história nacional, familiar e individual. Para essas pessoas, o retorno ao País natal é geralmente impossível ou severamente comprometido pela situação política e social da guerra ou do conflito.

Diante da dificuldade de retomar a vida do período pré-migratório e de retornar a seu País natal, concomitantemente ou posteriormente ao trabalho terapêutico é realizado um trabalho psicossocial, a fim de possibilitar a adaptação ao país e às mudanças no contexto social (novas constelações familiares, papéis sociais, status profissional, mudanças nas condições de saúde física e mental).

\section{Considerações Finais}

OSAPSIR, na condição de clínica de atendimento psicológico especializado, enquadra-se nas preocupações do MSSS e da Agência quanto ao fornecimento de um atendimento adequado ao conjunto da população, e quanto à acessibilidade e integração do serviço dentro da rede de saúde. Cabe acrescentar que programas como o SAPSIR procuram não apenas tornar acessíveis os serviços a uma população específica, mas, sobretudo, proporcionar as mesmas possibilidades de sucesso no tratamento e no alcance dos objetivos propostos pela intervenção que aqueles serviços oferecidos aos demais grupos da população.

O trabalho realizado nessa clínica especializada responde aos princípios gerais da psicologia clínica no âmbito da saúde, isto é, a intervenção psicológica com o objetivo de proporcionar uma melhor qualidade de vida no que se refere à saúde mental e aos princípios gerais da intervenção biopsicossocial, preconizados pela psicologia da saúde. Trata-se de uma clínica interdisciplinar, que se apoia no conhecimento das diversas áreas implicadas na intervenção propriamente dita, que proporciona uma mediação e possibilita uma transição e uma articulação com o meio médico (hospitais ou ambulatórios), com o meio psicossocial (centros de saúde, ONG, associações, escolas etc.) e com o meio social (através da atenção à família e a associações culturais). Além do trabalho clínico e social realizado pelo

584 Sapsir, deve igualmente ser lembrado que o serviço é um campo de formação dos alunos em estágio de psicologia, serviço social e saúde pública, além de se tratar de um campo de supervisão clínica e de pesquisa em psicologia.

O SAPSIR atende às principais orientações definidas pela APA para a psicologia da saúde, quais sejam, segundo Fischer e Tarquinio (2006, p.9):

Contribuir à prevenção, ao diagnóstico e ao tratamento da doença; estudar os fatores psicológicos, sociais, emocionais e cognitivos que atuam nos comportamentos de saúde e de doença; desenvolver os conhecimentos no domínio da etiologia das doenças e dos fatores que protegem a saúde.

A abrangência alcançada pelas intervenções realizadas no SAPSIR - sem esquecer aquelas feitas no âmbito da consultoria oferecida aos profissionais dos centros de saúde e dos serviços sociais - enfatiza os recursos individuais, como a capacidade de adaptação e o desenvolvimento de novas habilidades. Além disso, essa clínica constitui um lugar privilegiado para as compreensões culturais e individuais acerca da saúde e da doença (no que se refere à etiologia e ao tratamento), em consonância com as propostas da psicologia clínica e da psicologia da saúde - ou seja, um espaço de busca do bem-estar, por meio da articulação entre o indivíduo e o social.

\section{Referências}

Brasil. Ministério da Saúde. (2002). As cartas da promoção da saúde. Brasília: MS.

Castro, E. K. (2004). Psicologia da saúde X psicologia hospitalar: definições e possibilidades de inserção profissional. Psicologia Ciência e Profissão, 24 (3), 48-57.

Costa, R. (2007). Interdisciplinaridade e equipes de saúde. Mental, 5 (8), 107-124.

Devereux, G. (1967). Le renoncement à l'identité comme défense contre l'anéantissement. Revue Française de Psychanalyse, 31 (1), 101-142.

Devereux, G. (1972). Ethnopsychanalyse complémentariste. Paris: Flammarion.

Dimenstein, M. (1998). O psicólogo nas Unidades Básicas de Saúde: desafios para a formação e atuação profissionais. Estudos em Psicologia, 3 (1), 53-81.

Fischer, G. N. (2002). Traité de psychologie de la santé. Paris: Dunod.

Fischer, G. N., \& Tarquinio, C. (2002). Les concepts fondamentaux de la psychologie de la santé. Paris: Dunod. 
Freud, S. (1988). Estudos sobre a histeria (Breuer e Freud). Rio de Janeiro: Imago. (Originalmente publicada em 1893/1895).

Martins Borges, L., \& Pocreau, J.-B. (2009). Reconhecer a diferença: o desafio da etnopsiquiatria. Psicologia em Revista, 15 (1), 232-245.

Mesmin, C., \& Bâ, M. (1995). La langue maternelle comme outil thérapeutique dans la prise en charge des familles migrantes et de leurs enfants. In C. Mesmin (Org.), Psychothérapies des enfants de migrants (pp.21-40). Grenoble: La Pensée Sauvage.

Moro, M. R. (1998). Psychothérapie transculturelle des enfants de migrants. Paris: Dunod.

Ministère de la Santé et des Services Sociaux du Québec. (n.d.). Les Responsabilités de Gouvernance. Retrieved September 21,2009, from <http://www.msss.gouv.qc.ca/ sujets/organisation/ssss_enbref/index.php?aid=5>.

Nathan, T. (1986). La Folie des autres: traite d'ethnopsychiatrie clinique. Paris: Dunod.

Nathan, T. (1994). L'influence qui guérit. Paris: Éditions Odile Jacob.

Régis Regionale de la Santé et des Services Sociaux. (2005). Mandat. Retrieved on Septemper 13, 2009, from <http:// www.rrsss03.gouv.qc.ca/RR-profil-mandat. html>.

Ronzani, T., \& Rodrigues, M. (2006). O psicólogo na atenção primaria à saúde: contribuições, desafios e redirecionamentos. Psicologia: Ciência e Profissão, 26 (1),132-143.
Spink, M. J.P. (2007). Psicologia sociale saúde: práticas, saberes e sentidos. Petróplois: Vozes.

Streit, U. (1996). Les migrants: adaptation de la thérapie psychanalytique dans un contexte interculturel. In P. Doucet \&W. Reid (Orgs.), Lapsychothérapie psychanalytique: une diversité de champs cliniques (pp.378-394). Montreal: Gaetan Morin Éditeur.

Sturm, G. (2004). Le racisme et l'exclusion. In M. R. Moro, Q. La Noe \& Y. Mouchenik (Orgs.), Manuel de psychiatrie transculturelle (pp.265-278). Grenoble: La Pensée Sauvage.

Turcotte, N. (2009). Population immigrée recensée au Québec et dans les régions en 2006: caractéristiques générales. Recensement de 2006, Données ethnoculturelles. Québec: Gouvernement du Québec.

Ville de Québec. (2009). Portrait de la population immigrante de la ville de Québec: service do développement économique. Québec: Ville de Québec.

Tremblay, M. (2006). L'accessibilité et l'adaptation des services de santé et des services sociaux et psychosociaux aux personnes issues des communautés ethnoculturelles de la région de la Capitale-Nationale. Bilan 2002-2005 et Plan d'action régional 2006-2009. Québec: Agence de la Santé et des Services Sociaux de la Capitale-Nationale.

Recebido em: 16/11/2010

Aprovado em: 28/3/2012 